\title{
Evaluación de dos métodos de extracción de pectina de la cáscara de cacao (Theobroma cacao)
}

\section{Evaluation of two methods of pectin extraction from the cocoa shells (Theobroma cacao) \\ Extracción de pectina de cáscara de cacao.}

\author{
Yandry Javier Rengifo Alava Mg.(1) \\ Juan Carlos Macias Moreira Mg. ${ }^{(2)}$ \\ Sandra Isabel Mendoza Velez Mg.(3) \\ Diana Marianella Pincay Figueroa Mg. ${ }^{(4)}$
}

1 Pontificia Universidad Católica del Ecuador-Campus Chone. correo yandryr84@hotmail.com Chone, Ecuador.

${ }^{2}$ Profesional investigador autónomo, correo ceuss98@ hotmail.com, Chone, Ecuador.

${ }^{3}$ Profesional investigador autónomo, correo sandra_2381@ hotmail.com, Calceta, Ecuador.

${ }^{4}$ Universidad Católica Santiago de Guayaquil, correo dianapf2182@gmail.com Guayaquil, Ecuador.

Contacto: yandryr84@hotmail.com

\section{Resumen}

A partir de cáscaras de cacao de la variedad nacional EET103, aplicando dos métodos de extracción: hidrólisis ácida e hidrólisis enzimática se obtuvo pectina, cuyos rendimientos se compararon mediante t-Student para identificar el método más eficiente; adicionalmente se caracterizó por espectrofotometría infrarroja los grupos funcionales con los picos representativos y con estos valores determinar el grado de esterificación de las pectinas extraídas. La comparación de medias arrojó diferencias significativas $(p \leq 0,05)$ entre los métodos, determinando que la extracción ácida reportó rendimientos más altos. El análisis espectrofotómetro determinó áreas $\left(\mathrm{cm}^{-1}\right)$ en las pectinas de extracción ácida y enzimática de 1720,47 y 1727,98 respectivamente en los 
grupos ésteres, 1632,10 y 1600,99 en los grupos ácidos, comparados con 1741,07 y 1637,95 de la pectina comercial, el GE de la pectina fue de 51,31\% en ácida y 51,90\% en enzimática. Para concluir, el método de extracción de hidrólisis ácido obtuvo mayor rendimiento que el método de hidrólisis enzimático, los picos de caracterización de los grupos funcionales comparten similitudes con los picos de la pectina comercial; y el GE en ambos métodos están por encima del 50\%, colocándola como una pectina de alto metoxilo.

Palabras clave: Cacao, cáscara, hidrólisis ácida, hidrólisis enzimática, pectina, rendimiento.

\section{Abstract}

From cocoa shells of the national variety EET103, applying two methods of extraction: acid hydrolysis and enzymatic hydrolysis pectin was obtained, whose yields were compared by t-Student to identify the most efficient method; additionally was characterized by infrared spectrophotometry functional groups with representative peaks and with these values determine the degree of esterification of the extracted pectins. The comparison of averages showed significant differences $(\mathrm{p} \leq 0,05)$ between the methods, determining that acid extraction reported higher yields. The spectrophotometer analysis determined areas (cm-1) in the acid and enzymatic extraction pectins of 1720,47 and 1727,98 respectively in the ester groups, 1632,10 and 1600,99 in the acid groups, compared with 1741,07 and 1637,95 in the commercial pectin, the pectin GE was 51,31\% in acid and $51,90 \%$ in enzymatic. To conclude, the acid hydrolysis extraction method performed better than the enzymatic hydrolysis method, the characterization peaks of the functional groups share similarities with the peaks of the commercial pectin; and the GE in both methods is above 50\%, placing it as a high methoxyl pectin.

Key words: Cocoa, shell, acid hydrolysis, enzymatic hydrolysis, pectin, yield.

\section{Introducción}

Las cáscaras representan el $90 \%$ del fruto y son el principal producto de residuo de la industria cacaotera, por tanto, representa un grave problema. Este residuo se convierte en una fuente significativa de enfermedades cuando es usado como abono en las plantaciones (Suárez y Orozco, 2014).

Las cáscaras representan un alto porcentaje de desecho en relación a los granos contenidos en ellas, el manejo inadecuado que se le da a este desecho, sumado al problema que representa para los cultivos contaminándolos por ser el hábitat perfecto para la proliferación de hongos como: Phytophthora megakarya, P. capsici y P. citrophthora responsables de afectar al fruto en las plantaciones (Badrie, Bekele, Sikora y Sikora, 2015). 
Una amplia gama de industrias usa el grano del cacao como materia prima en la producción de diversos productos con fines alimenticios, farmacéuticos, entre otros, generándose una gran cantidad de residuos (cáscaras) que contaminan el medio ambiente (Bravo y Condo, 2015).

Romero, Ticono y Dávila (2015) expresaron que los residuos agroindustriales son materiales de gran importancia en la industria alimenticia, pues, con la aplicación apropiada de tecnologías alternativas, son capaces de generar subproductos como jarabes azucarados que se utilizan en la obtención de otros productos económicamente factibles como el bioetanol (p. 101).

La abundante información respecto a la extracción de pectinas y de sus usos demuestra la importancia que tiene este compuesto orgánico en la industria (alimenticia principalmente). La gran variedad de materias primas para su obtención y la diferencia en las metodologías implementadas para la misma dan fe de la posibilidad de ampliar el estudio sobre el tema (Betancourt y Llano, 2009).

Los hidrolizados de pectinas son muy empleados en industrias alimenticias, farmacéuticas y cosméticas por sus propiedades físicas y funcionales (espesante, gelificantes, texturizante, emulsificantes, retenedores de agua, adsorción de aceites, entre otros). En los últimos años, ha aumentado el interés en estudiar la modificación de la pectina. Una serie de grupos hidroxilo y carboxilo distribuidos a lo largo de la cadena principal, así como una cierta cantidad de azúcares neutros presentados como cadenas laterales, hacen que la pectina sea capaz de preparar un amplio espectro de derivados (Barazarte, Sangronis y Unai, 2008).

\section{Materiales y Métodos}

Métodos y Técnicas

\section{Variables Respuestas}

Y1: Rendimiento

\section{Métodos de Extracción}

\section{Método Hidrólisis Ácida}

Aza y Méndez citado por Del Aguila y Zegarra (2016) expresaron que la extracción de pectinas por hidrólisis ácida se llevó a cabo a temperaturas cerca de $\operatorname{los} 80^{\circ} \mathrm{C}$ por al menos una hora como se aprecia en la figura 1. Las pectinas consecutivamente se extrajeron y separaron de los desechos de diversos frutos mediante acidificación; se realizó usando ácidos como: el cítrico, clorhídrico, fosfórico, nítrico o sulfúrico; después de concentrarlas, se precipitaron con la adición de alcohol, se secó, se granularon y por último se tamizaron. La 
extracción de las trazas de pectinas después del proceso anterior se lo realizó en soluciones acuosas ácidas no sensibles al calcio.

\section{Método Hidrólisis Enzimática}

Mendoza, Jiménez y Ramírez (2017), describieron que en la técnica de extracción enzimática realizado a escala de laboratorio como se detalla en la figura 2, en un beakers de $1000 \mathrm{~mL}$, se agregaron $170 \mu \mathrm{L}$, del complejo enzimático comercial, por cada $200 \mathrm{~g}$ de material vegetal; de acuerdo a la ficha técnica de aplicación del fabricante, se utilizó un rango de 0,2 a $1 \mathrm{~kg}$, por cada tonelada de materia prima, por lo que, se calcularon para 200 g de cáscara de cacao, a una temperatura de entre 40 y $50^{\circ} \mathrm{C}$ y pH de 5 . Los tiempos de extracción fueron de 60 y 120 minutos, la precipitación de la pectina se realizó con etanol al 96\%, adicionando 80\% del volumen de la solución péctica, por 30 minutos; la pectina, se filtró en tela muselina y se secó en estufa, hasta obtener peso constante.

\section{Análisis Físico-Químicos}

Una vez obtenidos los mejores rendimientos de cada método de extracción se analizaron las características físico-químicas de las muestras.

\section{- Espectrofotometría Infrarroja (IR)}

Se tomó una muestra de cada una de las pectinas extraídas, se las colocaron en la base del lector del espectro infrarrojo, se activó el equipo y se tomaron los resultados de la lectura del software sobre la longitud de onda en cada muestra. El equipo que se utilizó para la obtención de los resultados en este análisis fue FT-IR, marca Thermo Scientific, modelo Nicolet iS10.

\section{- Grado De Esterificación (GE)}

El grado de esterificación (GE) de la pectina extraída por los dos métodos se determinaron por espectrofotometría de infrarrojo, donde se utilizó un FT-IR, marca Thermo Scientific, modelo Nicolet iS10. El espectro obtenido fue comparable en su perfil al de un espectro de pectina comercial. Para determinar el GE se utilizó la relación del área de la banda correspondiente a los grupos carboxilos y el área de los grupos esterificados, aplicando la ecuación propuesta por Rascón, Martínez, Carvajal, Martínez y Campa (2016).

$$
G E=\left(\frac{\mathrm{A}_{\mathrm{gce}}}{\mathrm{A}_{\mathrm{gce}}+\mathrm{A}_{\mathrm{gcne}}}\right) * 100
$$


Donde:

GE: Grado de Esterificación

Agce: Área de grupo carboxílico esterificados.

Agcne: Área de grupo carboxílico no esterificados.

Factores de Estudio

Extracción

- Hidrolisis Ácida

Factor a $(\mathrm{pH}) \quad$ Factor b (tiempo de cocción)
a1: 1,5
b1: $20 \mathrm{~min}$
a2: 2,5
b2: $30 \mathrm{~min}$

- Hidrólisis Enzimática

Factor c (Tiempo)

c1: $60 \mathrm{~min}$

c2: $120 \mathrm{~min}$
Factor d (Temperatura)

d1: $40^{\circ} \mathrm{C}$

$\mathrm{d} 2: 50^{\circ} \mathrm{C}$

Comparación de los Métodos

Factor a (Hidrólisis ácida)

Factor b (Hidrólisis enzimática)

\section{Tratamientos}

Tabla 1: Tratamientos para hidrólisis ácida (Combinación de los niveles de los factores)

\begin{tabular}{lll}
\hline Tratamientos & Código & Descripción \\
\hline T1 & $\mathrm{a} 1 \mathrm{~b} 1$ & $(\mathrm{pH} 1,5)(20 \mathrm{~min})$. \\
T2 & $\mathrm{a} 1 \mathrm{~b} 2$ & $(\mathrm{pH} 1,5)(30 \mathrm{~min})$. \\
T3 & $\mathrm{a} 2 \mathrm{~b} 1$ & $(\mathrm{pH} 2,5)(20 \mathrm{~min})$. \\
T4 & $\mathrm{a} 2 \mathrm{~b} 2$ & $(\mathrm{pH} 2,5)(30 \mathrm{~min})$. \\
\hline
\end{tabular}

Tabla 2: Tratamientos para hidrólisis enzimática (Combinación de los niveles de los factores)

\begin{tabular}{lll}
\hline Tratamientos & Código & Descripción \\
\hline T1 & c1d1 & $(60 \min ).\left(40^{\circ} \mathrm{C}.\right)$ \\
\hline
\end{tabular}




\begin{tabular}{lll}
\hline T2 & $\mathrm{c} 1 \mathrm{~d} 2$ & $(60 \mathrm{~min}).\left(50^{\circ} \mathrm{C}.\right)$ \\
T3 & $\mathrm{c} 2 \mathrm{~d} 1$ & $(120 \mathrm{~min}).\left(40^{\circ} \mathrm{C}.\right)$ \\
T4 & $\mathrm{c} 2 \mathrm{~d} 2$ & $(120 \mathrm{~min}).\left(50^{\circ} \mathrm{C}.\right)$ \\
\hline
\end{tabular}

\section{Diseño Experimental}

Como se muestra en la tabla 3, el experimento se desarrolló bajo un Diseño Completamente al Azar (DCA) en arreglo factorial A x B, con un total de 4 tratamientos por métodos y 3 repeticiones por tratamientos expuestos en las tablas 1 y 2. Los datos obtenidos fueron sometidos a los supuestos del ANOVA (normalidad Shapiro Wilk y homogeneidad Test de Levene) con un $\alpha=0,05$. Al cumplirse los supuestos se realizó el análisis de varianza con un nivel de confianza del $95 \%$ y la separación de medias por Tukey al mismo nivel de confianza para cada uno de los métodos de extracción. Finalmente, por medio de T-Student se compararon los métodos de extracción de pectina y se determinó el más eficiente, donde se utilizó el programa estadístico INFOSTAT versión libre 2018I.

Tabla 3: Esquema ADEVA

\begin{tabular}{ll}
\hline Fuente de variación & $\begin{array}{l}\text { Grados de } \\
\text { libertad }\end{array}$ \\
\hline Tratamientos & 3 \\
Factor a & 1 \\
Factor b & 1 \\
Interacción $\mathbf{a * b}$ & 1 \\
Error & 8 \\
Total & 11 \\
\hline
\end{tabular}

\section{Unidad Experimental}

Para este estudio se tomaron $200 \mathrm{~g}$ de cáscara cacao. Se recolectaron cáscaras de mazorcas maduras de cacao de la variedad tipo Nacional clon EET-103, las que fueron lavadas y trasladadas al laboratorio donde fueron procesadas.Resultados y Discusión

La comparación de medias mediante t de Student realizados para comparar los rendimientos en los dos métodos de extracción de pectina mostrados en la tabla 4 , se determina diferencia significativa $(\mathrm{p}<0,05)$ entre ambos métodos. 
El método ácido reportó un mayor porcentaje de rendimiento en relación al método enzimático. Mendoza et al. (2017), indican que a mayor concentración de enzimas el rendimiento de pectina es significativamente alto a los obtenidos con hidrólisis ácida. Esta diferencia se asocia a la posible insuficiencia en el tiempo de extracción. Sin embargo, la concentración de enzima se mantuvo constante durante esta, ya que el tiempo si fue adecuado, a pesar de que el rendimiento fue menor.

Tabla 4: Promedio de pectina extraída de cáscara de cacao por métodos ácido y enzimático

\begin{tabular}{|c|c|}
\hline \multirow{2}{*}{$\begin{array}{l}\text { Método } \\
\text { extracción }\end{array}$} & Pectina \\
\hline & Rendimiento (\%) \\
\hline Ácida & $6,33 \mathrm{a}$ \\
\hline Enzimática & $3,94 \mathrm{~b}$ \\
\hline $\mathbf{p}$ & 0,031 \\
\hline
\end{tabular}

Promedios con letras distintas en columna difieren según la prueba de t Student al 0,05 de error.

Como se muestra en la tabla 5, el efecto del pH sobre el rendimiento en la obtención de la pectina es significativo $(\mathrm{p}<0,05)$, sin embargo, el factor tiempo y la interacción con el pH no muestran significación.

A menor $\mathrm{pH}$ y mayor tiempo de hidrólisis se obtuvo mejor producción de pectina conforme a lo reportado por Maldonado, Salazar, Millones, Torres y Vásquez (2010), en su investigación donde el mayor rendimiento de pectina, se dio cuando emplearon el pH más bajo, coincidiendo con los resultados obtenido y descritos en el presente trabajo.

Tabla 5: Efectos de los factores y la combinación de ambos en el rendimiento de la pectina en la extracción ácida

\begin{tabular}{ll}
\hline Tratamientos & $\begin{array}{l}\text { Método Ácida } \\
\text { Pectina (\%) }\end{array}$ \\
\hline Efecto de $\mathbf{p H}$ & \\
\hline 1,5 & $8,37 \pm 0,76 \mathrm{a}$ \\
2,5 & $1,5 \pm 0,76 \mathrm{~b}$ \\
$\mathrm{p}$ & 0,0089 \\
\hline Efecto de Tiempo (min) & \\
\hline 30 & $7,45 \pm 0,76$ \\
20 & $5,22 \pm 0,76$ \\
\hline
\end{tabular}




\begin{tabular}{lcl}
\hline $\mathrm{p}$ & 0,0815 \\
\hline Efecto de interacción pH x tiempo $(\mathbf{m i n})$ & \\
\hline 1,5 & 30 & $10,21 \pm 1,07$ \\
1,5 & 20 & $6,53 \pm 1,07$ \\
2,5 & 30 & $4,69 \pm 1,07$ \\
2,5 & 20 & $3,91 \pm 1,07$ \\
$\mathrm{p}$ & & 0,2232 \\
\hline
\end{tabular}

Letras distintas en columna difieren según separación de medias tukey al 0,05 de error.

Valores medios terror estándar.

Lo expuesto en la tabla 6, muestra que los efectos del tiempo, la temperatura y la combinación de ambas, no reflejan diferencias significativas, sin embargo, el mayor tiempo de hidrólisis combinado con la mayor temperatura proporciona un rendimiento numéricamente representativo. Mendoza et al. (2017), indicaron que utilizando la mayor concentración de complejo enzimático obtuvo el mejor rendimiento en sus tratamientos, en contraste con la interacción de tiempos y temperatura que no reflejaron diferencias significativas.

Tabla 6: Efectos de los factores y la combinación de ambos en el rendimiento de la pectina en la extracción enzimática

\begin{tabular}{|c|c|c|}
\hline Tratamientos & & $\begin{array}{l}\text { Método Enzimática } \\
\text { Pectina }(\%)\end{array}$ \\
\hline \multicolumn{3}{|c|}{ Efecto de Tiempo (min) } \\
\hline 120 & & $4,57 \pm 0,97$ \\
\hline 60 & & $3,29 \pm 0,97$ \\
\hline $\mathrm{p}$ & & 0,3864 \\
\hline \multicolumn{3}{|c|}{ Efecto de Temperatura $\left({ }^{\circ} \mathrm{C}\right)$} \\
\hline 50 & & $4,36 \pm 0,97$ \\
\hline 40 & & $3,50 \pm 0,97$ \\
\hline $\mathrm{p}$ & & 0,556 \\
\hline \multicolumn{3}{|c|}{ Efecto de interacción tiempo $(\min ) \mathrm{x}$ temperatura $\left({ }^{\circ} \mathrm{C}\right)$} \\
\hline 120 & 50 & $5,53 \pm 1,37$ \\
\hline 120 & 40 & $3,62 \pm 1,37$ \\
\hline 60 & 40 & $3,39 \pm 1,37$ \\
\hline
\end{tabular}




\begin{tabular}{lll}
\hline 60 & 50 & $3,19 \pm 1,37$ \\
$\mathrm{p}$ & & 0,4724 \\
\hline
\end{tabular}

Valores medios terror estándar.

En la tabla 7, se muestra las diferencias numéricas de los picos característicos de la pectina obtenida en la investigación y los de la pectina comercial RAPID SED evaluada por Betancourt y Llano (2009). En las figuras 3, 4 y 5 se observan las longitudes de onda de la pectina extraídas por el método ácido, el método enzimático y la realizada por el autor antes mencionado a la pectina comercial en el espectro infrarrojo.

Tabla 7: Cuadro comparativo de los picos de longitud de onda en el espectro infrarrojo de las pectinas extraídas y la pectina comercial (rapid sed)

\begin{tabular}{llllll}
\hline \multirow{2}{*}{ Variantes } & \multicolumn{6}{l}{ Grupos funcionales } & & & \\
\cline { 2 - 6 } & $\begin{array}{l}\text { C=O } \\
\text { ÉSTER }\end{array}$ & $\begin{array}{l}\text { ĆCO } \\
\text { ÁCIDO }\end{array}$ & C-H & C-O & $-\mathbf{O H}$ \\
\hline $\begin{array}{l}\text { Pectina Ácida } \\
\left(\mathbf{c m}^{-1}\right)\end{array}$ & 1720,47 & 1632,10 & 2919,64 & 1013,40 & 3311,40 \\
$\begin{array}{l}\text { Pectina Enzimática } \\
\left(\mathbf{c m}^{-1}\right)\end{array}$ & 1727,98 & 1600,99 & 2927,03 & 1242,60 & 3262,40 \\
$\begin{array}{l}\text { Pectina comercial } \\
\left(\mathbf{c m}^{-1}\right)\end{array}$ & 1741,07 & 1637,95 & 2932,33 & 1070,00 & 3390,20 \\
\hline
\end{tabular}

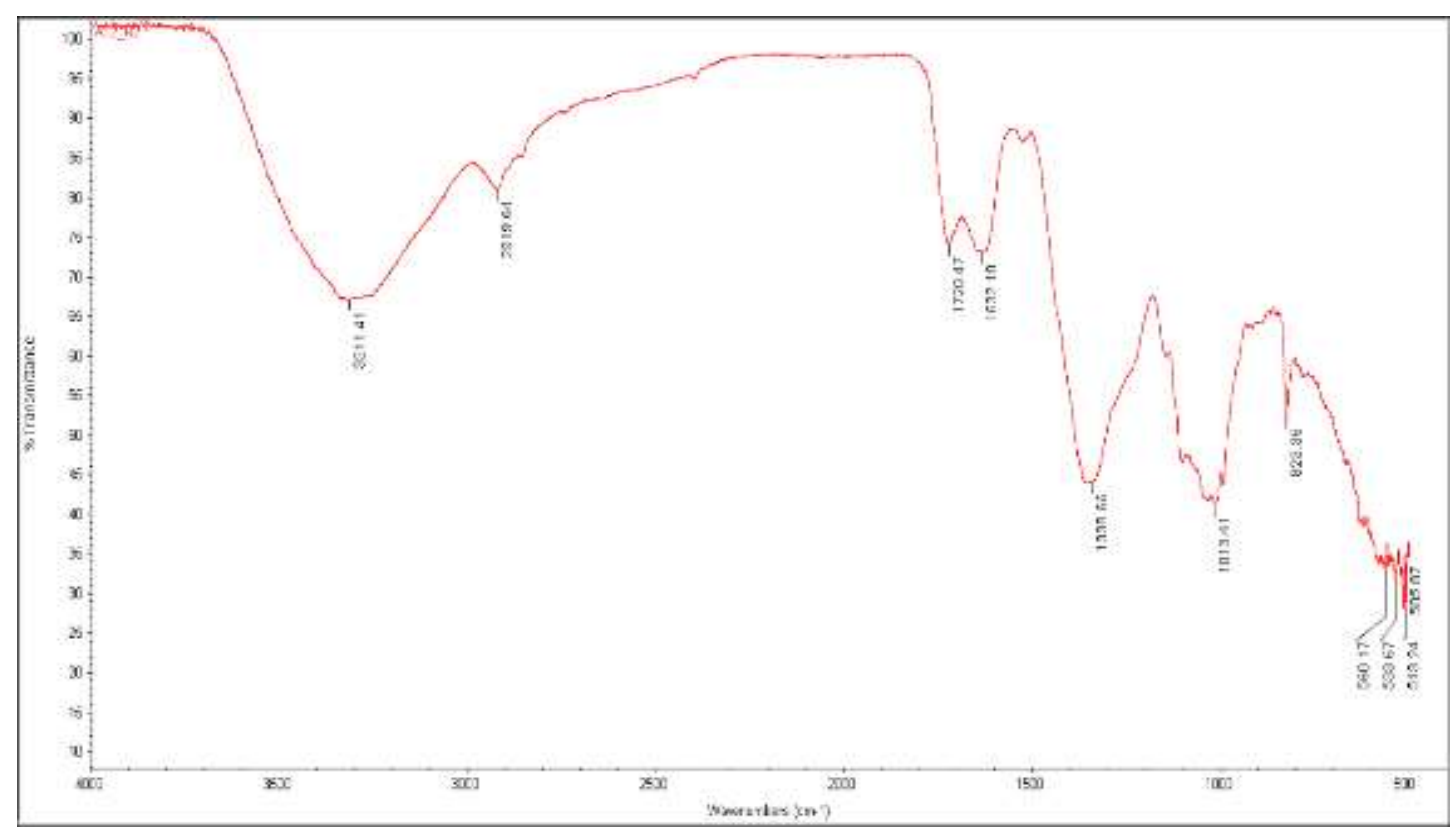

Figura 1: Longitud de onda del espectro IR en la pectina del método ácido 


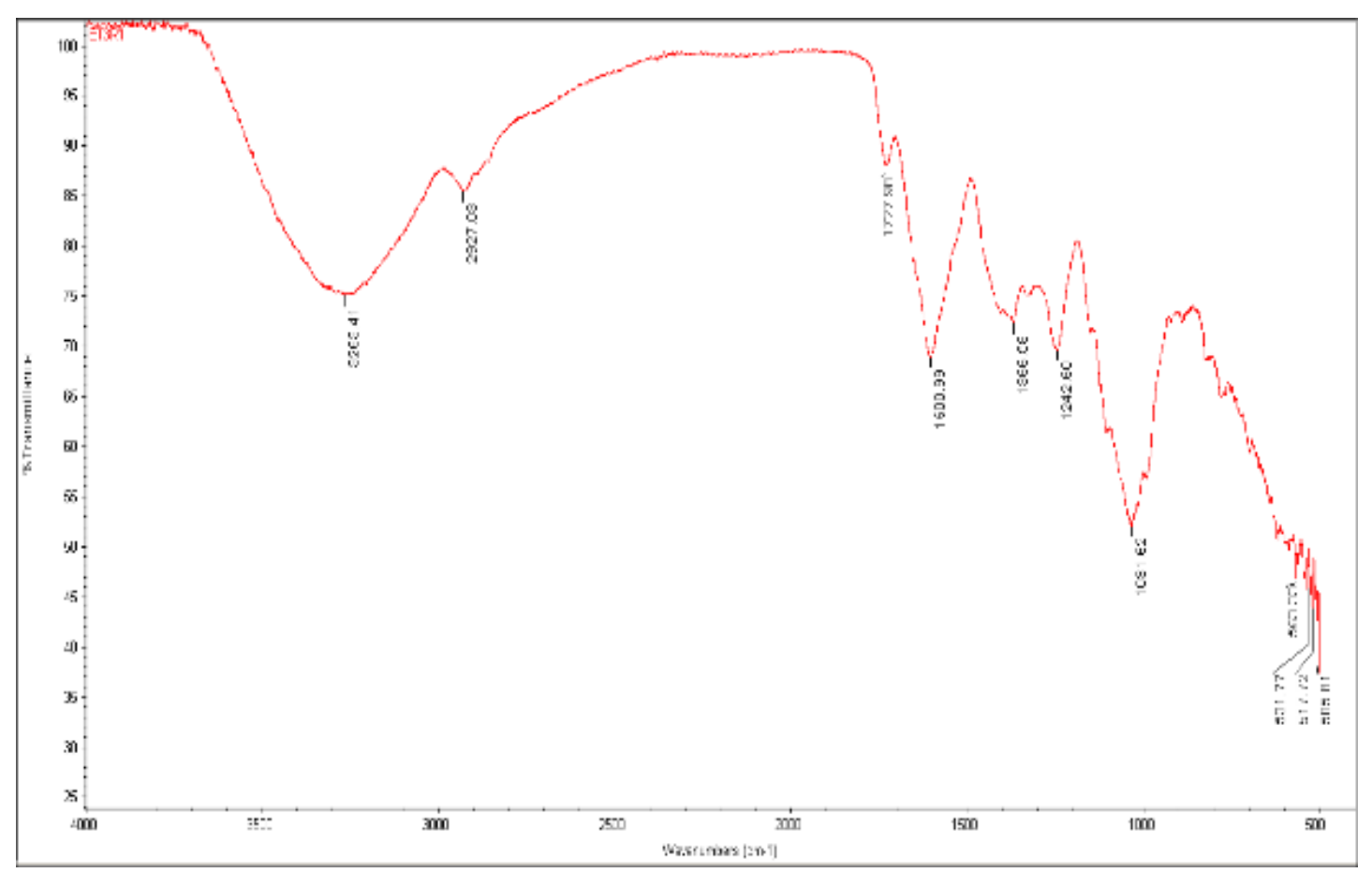

Figura 2: Longitud de onda del espectro IR en la pectina del método enzimático

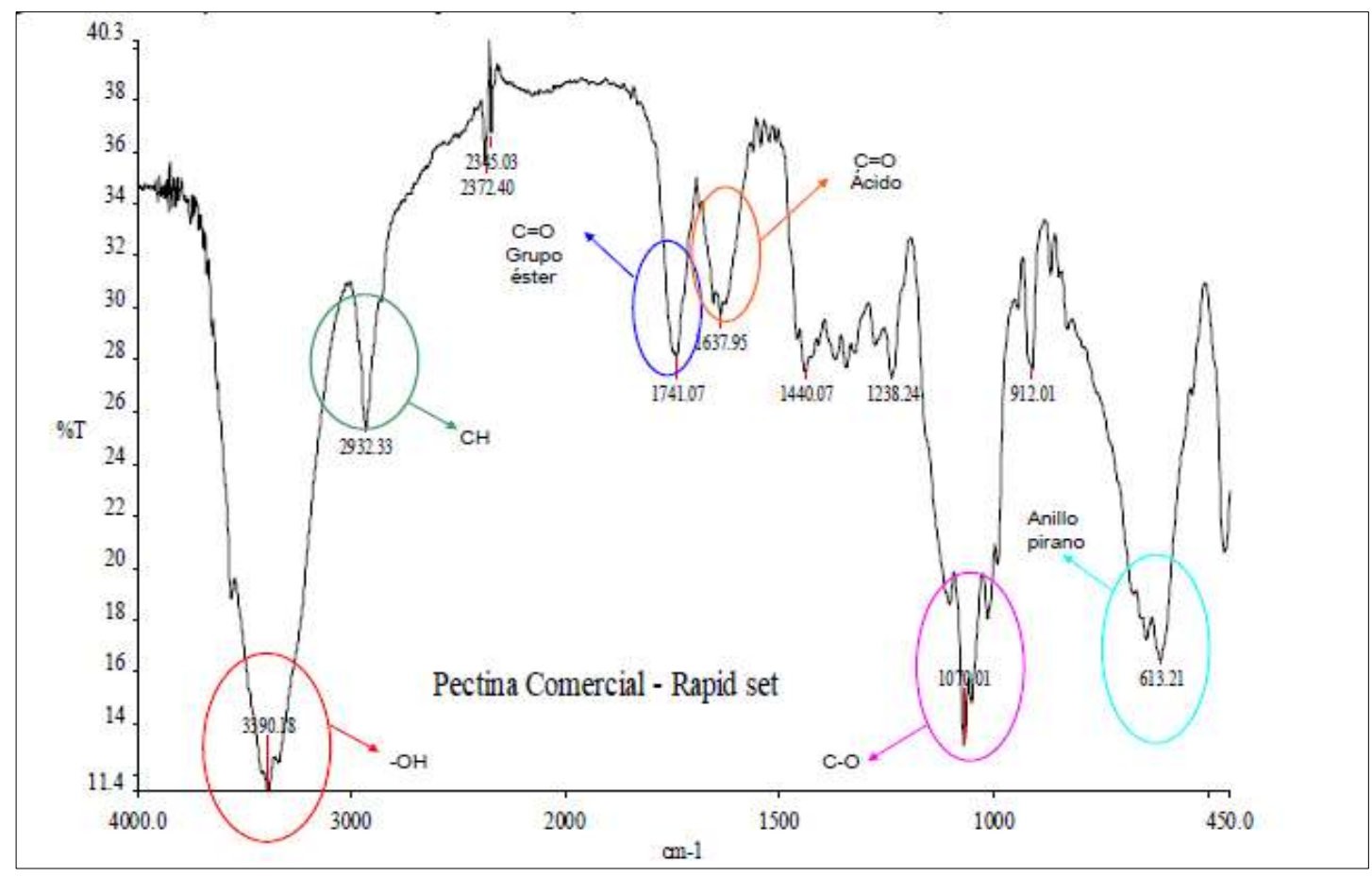

Figura 3: Longitud de onda del espectro IR en la pectina comercial Rapid set 
El grado de esterificación de la pectina extraída, por los métodos de hidrólisis ácida e hidrólisis enzimática fueron de 51,31 y 51,90\% respectivamente, lo cual nos indica que la pectina que se obtiene de la cáscara de cacao es de alto grado metoxilo, tal como expresa Rascón et al. (2016) en el trabajo realizado, el grado de esterificación de las pectinas extraídas en dos variedades de manzanas fue de 57 y $63 \%$ categorizándolas con pectinas de alto grado metoxilos e indicando que valores de esterificación por encima de $50 \%$ se consideran de alto grado.

\section{Conclusiones}

- $\quad$ El método de extracción hidrólisis ácida obtuvo un mayor rendimiento de pectina en relación con la hidrólisis enzimática.

- $\quad$ El área que ocupan los picos de los grupos carboxílicos esterificados y no esterificados de la longitud de onda de las pectinas extraídas por ambos métodos comparten similitudes con los picos de la pectina comercial.

- Ambos métodos de extracción dieron como resultado pectinas de alto grado metoxilo.

\section{Bibliografía}

Aza, E. \& Méndez, A. (2011). Extracción de pectina de Nopal (Opuntia Ficus indica) por medio ácido aplicando dos niveles de temperatura, tiempos y estados de madurez. (Tesis pregrado). Universidad Técnica del Norte. Tulcán, Ecuador. Recuperado de http://repositorio.utn.edu.ec/bitstream/123456789/743/2/03\%20AGI\%20293\%20ART\%C3\%8DCUL O\%20CIENT\%C3\%8DFICO.pdf.

Badrie, N., Bekele, F., Sikora, E. \& Sikor, M. (2012). Cocoa agronomy, quality, nutritional and health aspects. Critical Reviews in Food Science and Nutrition. 55(5), 620-659. doi:10.1080/10408398.2012.669428

Barazarte, H., Sangronis, E. \& Unai, E. (2008). Cocoa (Theobroma cacao) hulls: a posible commercial source of pectins. Archivos Latinoamericanos de Nutrición, 58(1), 64-70. Recuperado de http://www.scielo.org.ve/scielo.php?script=sci_arttext\&pid=S000406222008000100009.

Betancourt, L. \& Llano, J. (2009). Extracción de pectina a partir de los subproductos del beneficio del cacao., Universidad de EAFIT. Medellín Colombia. Recuperado de https://core.ac.uk/download/pdf/47237189.pdf.

Bravo, A. \& Condo, E. (2015). Comparación de la pectina obtenida a partir del aprovechamiento de las cáscaras de banano y cacao por el método de hidrólisis ácida (tesis pregrado). Universidad de Guayaquil, Guayaquil, Ecuador. Recuperado de http://repositorio.ug.edu.ec/handle/redug/8938.

Del Aguila, D. \& Zegarra, D. (2016). Extracción de pectina por hidrólisis ácida y precipitación alcohólica a partir de las cáscaras de cacao híbrido ccn51 (theobroma cacao 1.) para la fabricación de un prototipo 
de empaque alimentario, Pucallpa, región Ucayali 2015. (Tesis de pregrado). Universidad nacional intercultural de la Amazonia. Pucallpa, Perú. Recuperado de: http://repositorio.unia.edu.pe/bitstream/unia/103/1/TESIS.pdf

Maldonado, Y., Salazar, S., Millones, C., Torres, E. \& Vásquez. (2010). Extracción de pectina mediante el método de hidrólisis ácida en fruto de maushan (Vasconcellea weberbaueri (Harms) V.M. Badillo) proveniente del distrito de San Miguel de Soloco, región Amazonas. Revista Aporte Santiaguino; 3(2): 177-184. Recuperado de: http://www.scielo.org.pe/pdf/as/v3n2/a05v3n2.

Mendoza, L., Jiménez, J. \& Ramírez, M. (2017). Evaluación de la pectina extraída enzimáticamente a partir de las cáscaras del fruto de cacao (Theobroma cacao). Revista U.D.C.A Actualidad \& Divulgación Científica, 20(1), 131-138. $\quad$ Recuperado de http://www.scielo.org.co/scielo.php?script=sci_arttext\&pid=S012342262017000100015\&lng=en\&tln $\mathrm{g}=\mathrm{es} . \mathrm{es} / 2017 / 2 / \mathrm{art}-10 /$.

Rascón, A., Martínez, A., Carvajal, E., Martínez, K. \& Campa, A. (2016). Gelificación iónica de péctica de bajo grado de esterificación extraída de manzanas inmaduras de raleo. Rev. Fitotec. Mex., 39(1), $17-$ 24. Recuperado de: www.scielo.org. $\mathrm{mx} / \mathrm{pdf} / \mathrm{rfm} / \mathrm{v} 39 \mathrm{n} 1 / \mathrm{v} 39 \mathrm{n} 1 \mathrm{a} 5$.pdf.

Romero, H., Tinoco, O., \& Dávila, K. (2015). Hidrólisis enzimática de residuos agroindustriales del banano para la obtención de jarabe glucosado aplicando tres pretratamientos. Industrial Data, 18(1), 101-107. Recuperado de http://revistasinvestigacion.unmsm.edu.pe/index.php/idata/article/view/12072.

Suárez, D. \& Orozco, D. ( 2014). Obtención y caracterización de pectina a partir de la cascarilla de cacao del Theobroma cacao, subproducto de una industria chocolatera nacional., Universidad Tecnológica De Pereira. Colombia. Recuperado de http://www.academia.edu/28418197/OBTENCI\%C3\%93n_y_caracterizaci\%c3\%93n_de_pectina_a_ partir_de_la_cascarilla_de_cacao_del_theobroma_cacao_l._subproducto_de_una_industria_chocolate ra_nacional. 